\title{
Classical and Quantum Algorithms for Exponential Congruences
}

\author{
Wim van Dam ${ }^{1}$ and Igor E. Shparlinski ${ }^{2}$ \\ 1 Department of Computer Science, Department of Physics, University of California, \\ Santa Barbara, CA 93106-5110, USA \\ vandam@cs.ucsb.edu \\ 2 Department of Computing, Macquarie University, NSW 2109, Australia \\ igor@ics.mq.edu.au
}

\begin{abstract}
We discuss classical and quantum algorithms for solvability testing and finding integer solutions $x, y$ of equations of the form $a f^{x}+b g^{y}=c$ over finite fields $\mathbb{F}_{q}$. A quantum algorithm with time complexity $q^{3 / 8}(\log q)^{O(1)}$ is presented. While still superpolynomial in $\log q$, this quantum algorithm is significantly faster than the best known classical algorithm, which has time complexity $q^{9 / 8}(\log q)^{O(1)}$. Thus it gives an example of a natural problem where quantum algorithms provide about a cubic speed-up over classical ones.
\end{abstract}

\section{Introduction}

Let $\mathbb{F}_{q}$ be a finite field of $q$ elements and let $\mathbb{F}_{q}^{*}$ denote the multiplicative group of nonzero elements of $\mathbb{F}_{q}$. For $a, b, c, f, g \in \mathbb{F}_{q}^{*}$ we consider the equations

$$
a f^{x}+b g^{y}=c
$$

in nonnegative integers $x$ and $y$.

Equation (1) has a long history of study in number theory. In particular, it is dual closely related to the classical problem of finding $f, g \in \mathbb{F}_{q}$ for fixed $a, b$ and $x, y$ from the theory cyclotomic classes, see [2,11], which looks like a dual problem to studying Equation (1) but in fact, after a change of variables, become equivalent.

Furthermore, Equation (1) and variants of it also appeared in recent work of A. Lenstra and B. de Weger [8] and have been shown to be of cryptographic significance. In particular, the question about difficulty of finding solutions to Equation (1) has been discussed in [8] but now concrete results have been know before the present work.

In the theory of quantum computing the task of finding the solutions to Equation (1) is of importance when trying to solve the hidden subgroup problem for semi-direct product groups $\mathbb{Z} / N \rtimes \mathbb{Z} / p$ with $p=\Theta(\sqrt{N})$, 
see [1], where, as usual, $A=\Theta(B)$ means that $A=O(B)$ and $B=$ $O(A)$ (hereafter all implied constants are absolute). Furthermore it is also natural to consider this problem as a generalization of the discrete logarithm problem in $\mathbb{F}_{q}$, which can be solved efficiently using Shor's algorithm [10].

In this article we use some number theoretic tools to design classical and quantum algorithms that are more efficient than the brute force search (but unfortunately still have a running time exponential in the input size $\log q$ ). We use our classical algorithm to measure the level of improvement that can be achieved by allowing quantum algorithms. Ignoring $\log q$ terms, the classical algorithm that we present here has complexity $O^{*}\left(q^{9 / 8}\right)$ (which seems to be the best known) whereas we also present a quantum algorithm with complexity $O^{*}\left(q^{3 / 8}\right)$, where, as usual, $A=O^{*}(B)$ means that $A=B(\log B)^{O(1)}$. In particular, it gives an example of a natural problem where quantum algorithms provide an asymptotically cubic speed-up over classical ones.

Certainly if $f$ or $g$ is a primitive root, which generates all of $\mathbb{F}_{q}^{*}$, then the problem is not harder than the discrete logarithm problem. Moreover, in general our results suggest that finding solutions to Equation (1) becomes easier in case $f$ or $g$ is of large order, but still it appears to be much harder than the discrete logarithm problem.

\section{The Number of Solutions to the Equation}

\subsection{The Worst Case}

Here we use bounds of multiplicative character sums over finite fields to show that if the orders of $f$ and $g$ are large enough, then Equation (1) has a solution with at least one reasonably small component $x$ or $y$.

Lemma 1. Let $a, b, c \in \mathbb{F}_{q}^{*}$ and let $f$ and $g \in \mathbb{F}_{q}$ be of multiplicative orders $s$ and $t$, respectively. Then for any positive integer $r \leq t$, the equation $a f^{x}+b g^{y}=c$ has rs $/(q-1)+O\left(q^{1 / 2} \log q\right)$ solutions in nonnegative integers $x$ and $y$ with $x \in\{0, \ldots, s-1\}$ and $y \in\{0, \ldots, r-1\}$.

Proof. Let $k=(q-1) / s$ and let $\mathcal{X}_{k}$ be the group of all $k$ multiplicative characters $\chi: \mathbb{F}_{q} \rightarrow \mathbb{C}$ of order $k$, that is, $\chi^{k}=\chi_{0}$, the principal character, for any $\chi \in \mathcal{X}_{k}$ (see [9]). Note that for all non-empty $\mathcal{X}_{k}$ this group contains $k$ elements. For any $u \in \mathbb{F}_{q}$ we have

$$
\frac{1}{k} \sum_{\chi \in \mathcal{X}_{k}} \chi(u)= \begin{cases}1, & \text { if } u^{s}=1 \\ 0, & \text { otherwise }\end{cases}
$$


Noting that $u \in \mathbb{F}_{q}$ belongs to the group generated by $f$ if and only if $u^{s}=1$, we derive that the number $N_{a, b, c}(r, s)$ of solutions to Equation (1) with $x \in\{0, \ldots, s-1\}$ and $y \in\{0, \ldots, r-1\}$ equals

$$
N_{a, b, c}(r, s)=\sum_{y=0}^{r-1} \frac{1}{k} \sum_{\chi \in \mathcal{X}_{k}} \chi\left(a^{-1}\left(c-b g^{y}\right)\right) .
$$

Changing the order of summation and separating the term $r / k$ corresponding to the principal character $\chi_{0}$ we obtain

$$
\left|N_{a, b, c}(r, s)-\frac{r}{k}\right| \leq \frac{1}{k} \sum_{\chi \in \mathcal{X}_{k} \backslash\left\{\chi_{0}\right\}} \chi\left(a^{-1}\right) \sum_{y=0}^{r-1} \chi\left(c-b g^{y}\right) .
$$

By [12, Theorem 3] (see also [5]) each summation over $y$ is bounded by $O\left(q^{1 / 2} \log q\right)$ (provided $\left.1 \leq r \leq t\right)$, hence we have

$$
N_{a, b, c}(r, s)=\frac{r}{k}+O\left(q^{1 / 2} \log q\right),
$$

which concludes the proof.

From Lemma 1 we can immediately conclude the following.

Corollary 1. Let $a, b, c \in \mathbb{F}_{q}^{*}$ and let $f$ and $g \in \mathbb{F}_{q}$ be of multiplicative orders $s$ and $t$, respectively. There exists an absolute constant $C>0$ such that if for some integer $r$ we have

$$
C q^{3 / 2} s^{-1} \log q \leq r \leq t
$$

then the equation $a f^{x}+b g^{y}=c$ has a solution in integers $x$ and $y$ with $x \in\{0, \ldots, s-1\}$ and $y \in\{0, \ldots, r-1\}$.

We remark that the constant $C$ in Corollary 1 is independent of all variables involved ( $a, b, c, f, g$ and $q$ ) and that it is effectively computable. This result reduces the number of $(x, y)$ pairs that has to be searched for a solution to Equation (1). In Sections 3.1 and 4.1 we show how this reduction can be used to construct non-trivial worst case algorithms, both classical and quantum.

\subsection{The Typical Case}

To solve the equation $a f^{x}+b g^{y}=c$ for typical $c \in \mathbb{F}_{q}$ we now show that for almost all $c \in \mathbb{F}_{q}^{*}$ the results of Corollary 1 can be improved, which in turn will yield better average case algorithms for the central problem. 
Lemma 2. Let $a, b, c \in \mathbb{F}_{q}^{*}$ and let $f$ and $g \in \mathbb{F}_{q}$ be of multiplicative orders $s$ and $t$, respectively. For any positive integer $r \leq t$ and $\delta>0$, for all but $q / \delta^{2}$ elements $c \in \mathbb{F}_{q}^{*}$, the equation $a f^{x}+b g^{y}=c$ has $r s / q+\vartheta \delta \sqrt{r}$ solutions in nonnegative integers $x$ and $y$ with $x \in\{0, \ldots, s-1\}, y \in\{0, \ldots, r-1\}$ and $-1<\vartheta<1$.

Proof. Let $\psi: \mathbb{F}_{q} \rightarrow \mathbb{C}$ be a nontrivial additive character. We recall that for for any $u \in \mathbb{F}_{q}$ we have

$$
\frac{1}{q} \sum_{\lambda \in \mathbb{F}_{q}} \psi(\lambda u)= \begin{cases}1, & \text { if } v=0 \\ 0, & \text { if } v \in \mathbb{F}_{q}^{*}\end{cases}
$$

As in the proof of Lemma 1 we use $N_{a, b, c}(r, s)$ to denote the number of solutions to Equation (1) with $x \in\{0, \ldots, s-1\}$ and $y \in\{0, \ldots, r-1\}$. We have

$$
\begin{aligned}
N_{a, b, c}(r, s) & =\sum_{x=0}^{s-1} \sum_{y=0}^{r-1} \frac{1}{q} \sum_{\lambda \in \mathbb{F}_{q}} \psi\left(\lambda\left(a f^{x}+b g^{y}-c\right)\right) \\
& =\frac{s r}{q}+\frac{1}{q} \sum_{\lambda \in \mathbb{F}_{q}^{*}} \sum_{x=0}^{s-1} \sum_{y=0}^{r-1} \psi\left(\lambda\left(a f^{x}+b g^{y}-c\right)\right),
\end{aligned}
$$

which averaged over $c \in \mathbb{F}_{q}$ equals $s r / q$. To calculate the variance from its average, we look at the value defined by

$$
W_{a, b}(r, s)=\sum_{c \in \mathbb{F}_{q}}\left(N_{a, b, c}(r, s)-\frac{r s}{q}\right)^{2},
$$

which equals

$$
\begin{gathered}
\frac{1}{q^{2}} \sum_{c \in \mathbb{F}_{q}} \sum_{\lambda_{1}, \lambda_{2} \in \mathbb{F}_{q}^{*}} \sum_{x_{1}, x_{2}=0}^{s-1} \sum_{y_{1}, y_{2}=0}^{r-1} \psi\left(\lambda_{1}\left(a f^{x_{1}}+b g^{y_{1}}-c\right)+\lambda_{2}\left(a f^{x_{2}}+b g^{y_{2}}-c\right)\right) \\
=\frac{1}{q^{2}} \sum_{\lambda_{1}, \lambda_{2} \in \mathbb{F}_{q}^{*}} \sum_{x_{1}, x_{2}=0}^{s-1} \psi\left(a\left(\lambda_{1} f^{x_{1}}+\lambda_{2} f^{x_{2}}\right)\right) \sum_{y_{1}, y_{2}=0}^{r-1} \psi\left(b\left(\lambda_{1} g^{y_{1}}+\lambda_{2} g^{y_{2}}\right)\right) \times \\
\sum_{c \in \mathbb{F}_{q}} \psi\left(c\left(\lambda_{2}+\lambda_{1}\right)\right) .
\end{gathered}
$$


The inner sum over $c$ vanishes unless $\lambda_{1}=-\lambda_{2}$ (in which case it is $q$ ) and therefore

$$
\begin{aligned}
W_{a, b}(r, s) & =\frac{1}{q} \sum_{\lambda \in \mathbb{F}_{q}^{*}} \sum_{x_{1}, x_{2}=0}^{s-1} \psi\left(a \lambda\left(f^{x_{1}}-f^{x_{2}}\right)\right) \sum_{y_{1}, y_{2}=0}^{r-1} \psi\left(b \lambda\left(g^{y_{1}}-g^{y_{2}}\right)\right) \\
& =\frac{1}{q} \sum_{\lambda \in \mathbb{F}_{q}^{*}}\left|\sum_{x=0}^{s-1} \psi\left(a \lambda f^{x}\right)\right|^{2}\left|\sum_{y=0}^{r-1} \psi\left(b \lambda g^{y}\right)\right|^{2} \cdot
\end{aligned}
$$

It is well known that

$$
\left|\sum_{x=0}^{s-1} \psi\left(a \lambda f^{x}\right)\right|^{2} \leq q^{1 / 2}
$$

for example, this follows from [9, Theorem 8.78] taken with $k=1$ and $g^{0}, g^{1}, \ldots, g^{s-1}$ the impulse response sequence (it can also be derived from the bound of Gauss sums, see [9, Theorem 5.32]). Therefore

$$
W_{a, b}(r, s) \leq \sum_{\lambda \in \mathbb{F}_{q}}\left|\sum_{x=0}^{s-1} \psi\left(a \lambda f^{x}\right)\right|^{2}
$$

(note that we have added $\lambda=0$ into the last sum). We also have the straightforward equality

$$
\sum_{\lambda \in \mathbb{F}_{q}}\left|\sum_{y=0}^{r-1} \psi\left(b \lambda g^{y}\right)\right|^{2}=\sum_{\lambda \in \mathbb{F}_{q}}\left|\sum_{y=0}^{r-1} \psi\left(\lambda g^{y}\right)\right|^{2}=q r
$$

(essentially, this is Parseval's identity, i.e. we used the unitarity of the Fourier transformation over $\mathbb{F}_{q}$ on the characteristic vector of the set $\left.\left\{g^{0}, \ldots, g^{r-1}\right\}\right)$ and thus

$$
W_{a, b}(r, s)=\sum_{c \in \mathbb{F}_{q}}\left|N_{a, b, c}(r, s)-\frac{r s}{q}\right|^{2} \leq q r .
$$

Hence, for any $\delta>0$, the violation

$$
\left|N_{a, b, c}(r, s)-\frac{r s}{q}\right| \geq \delta \sqrt{r}
$$

holds for no more than $q / \delta^{2}$ values of $c \in \mathbb{F}_{q}^{*}$. 
Using $\delta=\sqrt{\log q}$ in Lemma 2, we see that for all but $q / \log q=o(q)$ elements $c \in \mathbb{F}_{q}^{*}$ the equation $a f^{x}+b g^{y}=c$ has $r s / q+\vartheta \sqrt{r \log q}$ solutions in $x \in\{0, \ldots, s-1\}, y \in\{0, \ldots, r-1\}$ with $-1<\vartheta<1$. Therefore we can immediately conclude the following.

Corollary 2. Let $a, b, c \in \mathbb{F}_{q}^{*}$ and let $f$ and $g \in \mathbb{F}_{q}$ be of multiplicative orders $s$ and $t$, respectively. If for some integer $r$ we have

$$
q^{2} s^{-2} \log q \leq r \leq t
$$

then for all but $o(q)$ elements $c \in \mathbb{F}_{q}^{*}$, the equation $a f^{x}+b g^{y}=c$ has a solution in integers $x$ and $y$ with $x \in\{0, \ldots, s-1\}$ and $y \in\{0, \ldots, r-1\}$.

\section{Classical Algorithms}

\subsection{Worst Case Classical Algorithm}

We start with a classical deterministic algorithm that is more efficient than brute search.

Theorem 1. Let $a, b, c, f, g \in \mathbb{F}_{q}^{*}$. One can either find a solution $x, y \in$ $\mathbb{Z}_{\geq 0}$ of the equation a $f^{x}+b g^{y}=c$ or decide that it does not have a solution in deterministic time $q^{9 / 8}(\log q)^{O(1)}$ on a classical computer.

Proof. Using a standard deterministic factorization algorithm, we factor $q-1$ and find the orders $s$ and $t$ of $f$ and $g$ in time $q^{1 / 2}(\log q)^{O(1)}$. Assume without loss of generality that $s \geq t$ (otherwise of the roles of $s$ and $t$ are reversed in the proof below). Let $C$ be the constant of Corollary 1 and define

$$
r=\left\lceil C q^{3 / 2} s^{-1} \log q\right\rceil .
$$

By Corollary 1, if $r \leq t$ then the central equation $a f^{x}+b g^{y}=c$ is solvable for $(x, y) \in\{0, \ldots, s-1\} \times\{0, \ldots, r-1\}$. Otherwise, if $r>t$, there may or may not be a solution with $(x, y) \in\{0, \ldots, s-1\} \times\{0, \ldots, t-1\}$. As a result, the following algorithm proves the theorem.

If $r \leq t$ then for every $y \in\{0, \ldots, r-1\}$ we evaluate $a^{-1}\left(c-b g^{x}\right)$ and then try to compute its discrete logarithm to base $f$, that is, an integer $x$ with $f^{x}=a^{-1}\left(c-b g^{y}\right)$, in deterministic time $s^{1 / 2}(\log q)^{O(1)}$, see [4, Section 5.3]. When found, the algorithm outputs $(x, y)$ and terminates. The condition $t \geq r$ and assumption $s \geq t$ implies for $s$ :

$$
s^{2} \geq s t \geq s r \geq C q^{3 / 2} \log q,
$$


which gives for the time complexity of this case

$$
r \cdot s^{1 / 2}(\log q)^{O(1)}=q^{3 / 2} s^{-1 / 2}(\log q)^{O(1)} \leq q^{9 / 8}(\log q)^{O(1)} .
$$

If $r>t$ we perform the same procedure for every $y \in\{0, \ldots, t-1\}$. If none of the $y$ yield a solution, the algorithm reports that the central equation has no solution. In this case, the condition $t<r$ implies that

$$
s t<s r \leq C q^{3 / 2} \log q
$$

and since $t \leq s$, the time complexity of this case is also bounded by

$$
t \cdot s^{1 / 2}(\log q)^{O(1)} \leq(s t)^{3 / 4}(\log q)^{O(1)} \leq q^{9 / 8}(\log q)^{O(1)},
$$

which completes the proof.

It is natural to ask whether one can design a faster probabilistic algorithm. For some fields this is indeed possible due to the existence of subexponential algorithms for computing discrete logarithms, see [4, Section 6.4]. However in general probabilistic algorithms do not seem to give any significant advantage for our problem.

\subsection{Typical Case Classical Algorithm}

Similarly, using Corollary 2 instead of Corollary 1 and repeating the arguments of the proof of Theorem 1 with

$$
r=\left\lceil q^{2} s^{-2} \log q\right\rceil
$$

we obtain that for almost all $c$ a stronger result than Theorem 1 holds.

Theorem 2. Let $a, b, c, f, g \in \mathbb{F}_{q}^{*}$. For all but o $(q)$ elements $c \in \mathbb{F}_{q}^{*}$, one can either find a solution $x, y \in \mathbb{Z}_{\geq 0}$ of the equation $a f^{x}+b g^{y}=c$ or decide that it does not have a solution in deterministic time $q(\log q)^{O(1)}$ on a classical computer.

\section{Quantum Algorithms}

\subsection{Worst Case Quantum Algorithms}

On a quantum computer one has the advantage that calculating discrete logarithms can be done efficiently in time $(\log q)^{O(1)}$. In combination with the quadratic speed-up of quantum searching this gives the following quantum algorithm for the central problem. We start with an algorithm that works for any $f$ and $g$. 
Theorem 3. Let $a, b, c, f, g \in \mathbb{F}_{q}^{*}$. One can either find a solution $x, y \in$ $\mathbb{Z}_{\geq 0}$ of the equation $a f^{x}+b g^{y}=c$ or decide that it does not have a solution in time $q^{3 / 8}(\log q)^{O(1)}$ on a quantum computer.

Proof. We use Shor's algorithm [10] to compute $s$ and $t$ in polynomial time. Without loss of generality we assume that $s \geq t$ and we define $r$ by Equation (2) as in the proof of Theorem 1. A polynomial time quantum subroutine $\mathcal{S}(y)$ is constructed that, using Shor's discrete logarithm algorithm [10], for a given $y$ either finds and returns the integer $x$ with $f^{x}=a^{-1}\left(c-b g^{x}\right)$ or reports that no such $x$ exists.

If $r \leq t$, then, using Grover's search algorithm [6], we search the subroutines $\mathcal{S}(y)$ for all $y \in\{0, \ldots, r-1\}$ in time

$$
r^{1 / 2}(\log q)^{O(1)}=q^{3 / 4} s^{-1 / 2}(\log q)^{O(1)} \leq q^{3 / 8}(\log q)^{O(1)} .
$$

If $r>t$, we search the $\mathcal{S}(y)$ for all $y \in\{0, \ldots, t-1\}$ in time

$$
t^{1 / 2}(\log q)^{O(1)} \leq(s t)^{1 / 4}(\log q)^{O(1)} \leq q^{3 / 8}(\log q)^{O(1)} .
$$

As in the proof of Theorem 1, we conclude that due to our choice of $r$ we either find a solution to Equation (1) or conclude that there is no solution.

We now show that if $f$ and $g$ are of large order then there is a more efficient quantum algorithm.

Theorem 4. Let $a, b, c, f, g \in \mathbb{F}_{q}^{*}$ and let $f$ and $g$ be of multiplicative orders $s$ and $t$, respectively. There is an absolute constant $C$ such that if

$$
\text { st }>C q^{3 / 2}(\log q)^{1 / 2}
$$

then one can either find a solution $x, y \in \mathbb{Z}_{\geq 0}$ of the equation $a f^{x}+b g^{y}=c$ or decide that it does not have a solution in time $q^{1 / 2}(s t)^{-1 / 4}(\log q)^{O(1)}$ on a quantum computer.

Proof. Assume without loss of generality that $s \geq t$. It follows from the condition of the theorem and Lemma 1 that for some appropriate constant $C$ and

$$
r=\left\lfloor C q^{3 / 2} s^{-1}(\log q)^{1 / 2}\right\rfloor \leq t
$$

there are

$$
\frac{r s}{q-1}+O\left(q^{1 / 2} \log q\right) \geq \frac{r s}{2 q}
$$

solutions to Equation (1) with $x \in\{0, \ldots, s-1\}$ and $y \in\{0, \ldots, r-1\}$. 
We now use the version of Grover's search algorithm as described in [3] that finds one out of $m$ matching items in a set of size $r$ using only $O(\sqrt{r / m})$ queries. Here we search the subroutines $\mathcal{S}(y)$ for all $y \in$ $\{0, \ldots, r-1\}$ with the promise (which follows from Lemma that there are $m=r s /(q-1)+O\left(q^{1 / 2} \log q\right)$ solutions $(x, y)$. Because for each value $y$ there can be at most one value $x \in\{0, \ldots, s-1\}$ such that $a f^{x}+b g^{y}=c$ there are $m$ different values $y$ for which $\mathcal{S}$ will report a solution $x$, hence a solution will be found in time

$$
(r / m)^{1 / 2}(\log q)^{O(1)}=q^{1 / 2} s^{-1 / 2}(\log q)^{O(1)} .
$$

Since $s \geq(s t)^{1 / 2}$, this concludes the proof.

In particular, the running time of the algorithm of Theorem 4 is upper bounded by $O\left(q^{1 / 8}(\log q)^{O(1)}\right)$.

\subsection{Typical Case Quantum Algorithms}

Similarly to the classical case, for almost all $c \in \mathbb{F}_{q}$ stronger results than those of Theorems 3 and 4 are possible. For example, defining again $r$ by Equation (3) and arguing as in the proof of Theorem 3, we obtain the following result.

Theorem 5. Let $a, b, c, f, g \in \mathbb{F}_{q}^{*}$. For all but $o(q)$ elements $c \in \mathbb{F}_{q}^{*}$, one can either find a solution $x, y \in \mathbb{Z}_{>0}$ of the equation $a f^{x}+b g^{y}=c$ or decide that it does not have a solution in time $q^{1 / 3}(\log q)^{O(1)}$ on a quantum computer.

Finally, taking

$$
r=\left\lfloor q^{2} s^{-2} \log q\right\rfloor
$$

and using Lemma 1 in the argument of the proof of Theorem 4, we see that for almost all $c \in \mathbb{F}_{q}$ the complexity estimate of Theorem 4 holds for a wider range of $s$ and $t$.

Theorem 6. Let $a, b, c, f, g \in \mathbb{F}_{q}^{*}$ and let $f$ and $g$ be of multiplicative orders $s$ and $t$, respectively. For all but $o(q)$ elements $c \in \mathbb{F}_{q}^{*}$, if

$$
s t>q^{4 / 3}(\log q)^{2 / 3}
$$

then one can either find a solution $x, y \in \mathbb{Z}_{\geq 0}$ of the equation $a f^{x}+b g^{y}=c$ or decide that it does not have a solution in time $q^{1 / 2}(s t)^{-1 / 4}(\log q)^{O(1)}$ on a quantum computer. 


\section{Connection with the Hidden Subgroup Problem}

The pretty good measurement approach [1] to the Hidden Subgroup Problem (HSP) over the non-abelian group $\mathbb{Z} / q \rtimes \mathbb{Z} / p$ with $q$ a prime and $q / p^{2}=(\log q)^{O(1)}$ shows that the HSP can be solved efficiently on a quantum computer if one can efficiently solve the equation $a f^{x}+b f^{y}=c$, where $f$ has multiplicative order $p$ in $\mathbb{Z} / q$. All algorithms presented in this article have superpolynomial complexity in $\log q$ and hence fall short of this goal.

For this restricted problem with $f=g$ and $f$ of order $p \approx \sqrt{q}$, there are $p^{2}$ possible solutions $(x, y)$, hence even a classical algorithm has $O^{*}(q)$ time complexity instead of the $O^{*}\left(q^{9 / 8}\right)$ of Theorem 1 . Quantum mechanically, one can 'Grover search' the set of solutions $x \in\{0, \ldots, p-1\}$ in time $O^{*}\left(q^{1 / 4}\right)$, which, although better than the $O^{*}\left(q^{3 / 8}\right)$ of Theorem 3 , is still far from polynomial in $\log q$.

\section{Remarks and Open Problems}

We remark that in some finite fields classical subexponential probabilistic algorithms are possible for the discrete logarithm problem, see [4, Section 6.4]. In such fields, a version of Theorem 1 can be obtained with an algorithm that runs in probabilistic time $q^{3 / 4+o(1)}$, which is still much slower that the quantum algorithm of Theorems 3 and 4 . We note that although over the last several years fast heuristic algorithms for the discrete logarithm problem have been designed to work over any finite field, rigorous subexponential algorithms are know only for fields of special types (such as prime fields $\mathbb{F}_{p}$ or binary fields $\mathbb{F}_{2^{m}}$ ), see [4, Section 6.4$]$ for more details. Clearly using probabilistic algorithms one can also get additional speed up in the classical case if the multiplicative orders $s$ and $t$ are large (similar to Theorems 4 and 6).

To try to strengthen the presented results one can consider the analogue to Equation (1) for elliptic curves $\mathbb{E}$ over $\mathbb{F}_{q}$. For example, given two $\mathbb{F}_{q}$-rational points $F, G \in \mathbb{E}\left(\mathbb{F}_{q}\right)$ and the values $a, b, c \in \mathbb{F}_{q}$ one can ask for solutions to the equation

$$
a \cdot x([u] F)+b \cdot x([v] G)=c
$$

in integers $u$ and $v$ (where $x(Q)$ denotes the $x$-coordinate of a point $Q \in \mathbb{E}\left(\mathbb{F}_{q}\right)$ in a fixed affine model of $\mathbb{E}$ and $[n] Q$ denotes the $n$-fold sum $Q \oplus Q \oplus \cdots \oplus Q$ in the group of $\mathbb{E})$. Using bounds of character sums over subgroups of elliptic curves, see [7], one can obtain full analogues of our 
results (in fact at the cost of only typographical changes). This case is interesting since in the classical scenario even heuristic subexponential algorithms are not known.

But above of this all, it still remains an open problem whether or not there exist efficient quantum algorithms that run in time $(\log q)^{O(1)}$ for the determining the integer solutions $x, y$ to the equation $a f^{x}+b g^{y}=c$ and even the more restricted version $a f^{x}+b f^{y}=c$ over $\mathbb{F}_{q}$.

Acknowledgments. The authors are grateful to Michele Mosca for useful and encouraging discussions.

This work was initiated during a very pleasant visit by I.S. to the University of California at Santa Barbara whose hospitality is gratefully acknowledged. W.v.D. is supported by the Disruptive Technology Office (DTO) under Army Research Office (ARO) contract number W911NF-04R-0009 and the NSF CAREER award no. 0803963; I.S. is supported by ARC grant DP0556431.

\section{References}

1. D. Bacon, A. M. Childs and W. van Dam, From optimal measurement to efficient quantum algorithms for the hidden subgroup problem over semidirect product groups, Proceedings of the 46th Annual IEEE Symposium on Foundations of Computer Science (FOCS'05), 2005, pp. 469-478.

2. B. Berndt, R. Evans and K. S. Williams, Gauss and Jacobi Sums, Canadian Mathematical Society Series of Monographs and Advanced Texts, Volume 21, John Wiley \& Sons, 1998.

3. M. Boyer, G. Brassard, P. Høyer and A. Tapp, Tight bounds on quantum searching, Fortschritte der Physik, Vol. 46 (1998), pp. 493-505.

4. R. Crandall and C. Pomerance, Prime numbers: A computational perspective, Springer-Verlag, Berlin, 2005.

5. E. Dobrowolski and K. S Williams, An upper bound for the sum $\sum_{n=a+1}^{a+H} f(n)$ for a certain class of functions $f$, Proceedings of the American Mathematical Society, Vol. 114 (1992), pp. 29-35.

6. L. Grover, A fast quantum-mechanical algorithm for database search, Proceedings of the 28th Annual ACM Symposium on Theory of Computing (STOC'96), 1996, pp. $212-219$.

7. D. R. Kohel and I. E. Shparlinski, Exponential sums and group generators for elliptic curves over finite fields, Lecture Notes in Computer Science, Springer-Verlag, Berlin, Vol. 1838 (2000), pp. 395-404.

8. A. Lenstra and B. de Weger, On the possibility of constructing meaningful hash collisions for public keys, Lecture Notes in Computer Science, Springer-Verlag, Berlin, Vol. 3574 2005, pp. 267-279.

9. R. Lidl and H. Niederreiter, Finite Fields, Encyclopedia of Mathematics and Its Applications, Volume 20, Cambridge University Press, Cambridge, 1997. 
10. P. Shor, Polynomial-time algorithms for prime factorization and discrete logarithms on a quantum computer, SIAM Journal on Computing, Vol. 26 (1997), pp. 1484-1509.

11. T. Storer, Cyclotomy and Difference Sets, Lectures in Advanced Mathematics, Markham Publishing Company, 1967.

12. H. B. Yu, Estimates of character sums with exponential function, Acta Arithmetica, Vol. 97 (2001), pp. 211-218. 